\title{
DL-Arginine Hydrochloride
}

National Cancer Institute

\section{Source}

National Cancer Institute. DL-Arginine Hydrochloride. NCI Thesaurus. Code C62668.

The hydrochloride salt form of the amino acid DL-arginine, a racemic mixture of arg inine with potential chemopreventive properties. The mechanism of action by which DLarginine exert its chemopreventive effect may by be attributed to enhancement of detoxification enzymes, including the activation of certain antioxidant enzymes, which may lead to a reduction in the formation of free radicals. 